\title{
A Study on the Influence of Haze on Respiratory System of Outdoor Athletes

\author{
Jia Liu ${ }^{1, a}$ \\ ${ }^{1}$ Shaanxi Xueqian Normal University, Xi'an City, Shaanxi Province, 710100, China \\ aemail:453557763@qq.com
}

\section{Keywords: Haze; PM 2.5; Respiratory diseases; Particulates; Toxicity}

\begin{abstract}
Haze pollution is one of the most serious environmental pollution problems in modern society. There is a growing body of evidence that outdoor breathing exercises are more damaging to the respiratory system of people exposed to haze than those without exercise. In other words, sports disadvantages outweigh the benefits under the pollution of the haze weather. Researchers have found that dust that can affect the human respiratory system is a particle equivalent to less than 2.5 microns in diameter - PM 2.5. Therefore, this paper analyzed the influence of haze on the respiratory system of outdoor athletes based on PM 2.5.
\end{abstract}

\section{Introduction}

PM 2.5 refers to particles in the atmosphere, which is smaller than or equal to 2.5 microns in diameter and also known as accessible particulates mainly derived from fossil fuel combustion; the second is the two particles formed by chemical reactions, such as automobile exhaust gas and cigarette smoke. Although PM 2.5 has little content in the composition of the earth's atmosphere, it has an important bearing on air quality and visibility. Compared with coarse atmospheric particles, PM 2.5 has small particle size and large specific surface area; its diameter is less than 1 / 20 of human hair, and its shape and composition are quite complex. PM 2.5 not only contains a lot of organic matter such as benzopyrene, but also has many heavy metals such as plumbum $(\mathrm{Pb})$, nickel $(\mathrm{Ni})$, chromium $(\mathrm{Cr})$; because of its small gravity, it stays long in the atmosphere and has far distance.

The physiological structure of the human body determines that there is almost no filtering or blocking capability to the PM 2.5. As a result, PM 2.5 can enter the body with human respiration. It can get into the alveoli and deposit, interfere with the gas exchange and blood circulation of the lung, and lead to heart lung dysfunction and other related diseases. PM 2.5 is easy to settle in the respiratory tract and alveoli, causing large range of pollution and harms to human health. At the same time, these fine particles contain harmful gases, heavy metals and other dissolved in the blood, which brings more harms to human health. The effects of PM 2.5 on respiratory system mainly include three aspects: inflammatory injury, oxidative damage and mutagenicity, carcinogenic toxicity.

\section{Inflammatory Lesions}

It is difficult for the fine particles to contact the lung tissue cells, and it is difficult to drop. The lung tissue cells, especially alveolar macrophages (AM), are damaged by stimulation. Pulmonary macrophages play an important role in the local clearance of respiratory tract, and they are the main target cells of fine particles. Automobile exhaust particulates inhibit the immune function of AM, reduce the expression of FC receptors, and inhibit the cytotoxicity of tumor cells and antibody mediated 
cytotoxicity (ADCC) to a certain extent. The deposited fine particles act on type II alveolar epithelial cells, inhibit cell differentiation and cell metabolism, damage epithelial cells, and even lead to hyperplasia of lung epithelial cells and fibrosis. Churg found that the fine particles through the stimulation of platelet-derived growth factor (PDGF) and transforming growth factor alpha (TGF- alpha, beta, beta) which indirectly promotes epithelial and stromal hyperplasia, fibrous tissue caused by airway wall thickening, leading to inflammation.

Fine particles are mainly induced inflammation by the changes in biochemical components of lung tissue through adsorbing toxic components and releasing inflammatory factors. After injecting particulate matter into rat trachea, the content of lactate dehydrogenase (LDH), acid phosphatase (ACP) and sialic acid (NA) increased significantly in lung lavage fluid (BALF). Xiao Chunling also confirmed that fine particles can cause the increase of LDH and NA in rat BALF, both of which can be used as early sensitive indicators of lung parenchymal cell injury. Veronesi and other studies have found that $\mathrm{Ca} 2+$ is elevated in BEAS-2B cells exposed to fine particulate extracts, and the release of key inflammatory factors such as IL-6, IL-8, and TNF- alpha was increased. This suggests that the acidic soluble components of particulate matter stimulate the release of cytokines by Capsaicin and PH sensitive receptor pathways in BEAS-2B cells. PM 2.5 and carbon black particles act on RAW264.7 cells respectively. The level of TNF- alpha produced by PM 2.5 was 10 times that of carbon black particles, and the increase in IL-6 levels was observed. Carbon black particles do not have this effect, which indicates that the adsorption of pollutants on fine particles is the main reason for the release of inflammatory factors. But some studies have also found that the role of IL-6, TNF-, MCP-1 caused by particulate matter in lung tissue is derived fromPM 10. The ability of PM 10 to cause cytokine production is far greater than that of PM 2.5, so further studies are needed.

One pathway of induction of airway inflammatory factors by fine particles is by enhancing transcription levels of genes encoding transcription factors. Aamet found that diesel exhaust particles (ROFA) exposure leads to activation of the MAP kinase pathway, and increases the activity and quantity of the transcription factors (NF-Kb) and activating transcription factor 2 (ATF2) activity, which suggested that activation of transcription factors are associated with pulmonary inflammation and the increasing of the inflammation factor caused by it. NF-Kb is thought to play an important role in the inflammatory effects of fine particle mediated, and it is one of the factors regulating inflammatory gene transcription. In vitro experiment, the alveolar epithelial cells exposed to PM 2.5 in suspension, which can detect an increased NF- Kb regulate target gene transcription activity. It suggested that PM 2.5 may stimulate the activation of NF-Kb and effect on the target gene and induced gene expression. Fine particles enter the respiratory system and stimulate the secretion of cytokines;cytokines make synergistic effect on the activation of NF-Kb and initiate a range of related gene expression and produce cytokine cascade amplification effect, so that to cause widespread inflammatory lesions in the body.

Asthma is a chronic inflammatory disease of the airways associated with genetic and environmental factors. Atmospheric PM 2.5 pollution may be the cause of the asthma. PM 2.5 can be caused by the acute asthma respiratory stimulation. PM 2.5 can also act as a foreign substance to induce phagocytosis of bronchial mucosa and alveolar macrophages (AM) and produce an immune toxic effect, such as inhibition of type Thl lymphocytes, decreased IL-2 activity, elevated specific IgE under prolonged exposure. IgE binds to the mast cell surface of the airways, causing the body to become sensitized, and re-exposure to PM 2.5 causes asthma attacks. 


\section{Oxidative Damage}

Studies show that particulate matter has free radical activity, and that the metal components and organic components of fine particles can stimulate alveolar macrophages to produce free radicals and cause further damage to the tissues and cells. The iron, copper, zinc, manganese and other transition metals such as polycyclic aromatic hydrocarbons and lipopolysaccharide, which are rich in PM2.5 surface, can increase the formation of free radicals in lung. At the same time, an antioxidant component is consumed to initiate oxidative stress. Free radicals are harmful chemicals with strong oxidizing properties in the oxidation system. Once free radicals are generated, they can be expanded by intermediate metabolites (such as neutrophils) to form new radicals and form a chain reaction.

The damage of free radicals to lung tissue cells mainly has the following points: first, free radicals can damage the normal structure of alveolar epithelial cell membrane and it also can target the surface of alveolar cells and cause structural damage of alveolar epithelial cells and phagocytosis of lung macrophages; second, the free radicals start pulmonary and systemic inflammation through the activation of nuclear factor-Kb, upregulation of chemokine and inflammatory mediators. Third, free radicals can cause chromosome aberrations of nucleic acid changes or DNA fracture, interference of mitochondrial function and the initiation of programmed cell death. In addition, oxidative stress leads to intracellular calcium overload in sustained activation of nuclear factor-Kb, inflammatory reaction intensified or prolonged, or even develop into chronic inflammation.

Aust and other studies have found that the transition metals in fine particles cause oxidative stress and release of inflammatory mediators in the lung epithelial cells (A549). Hirano shows the organic components of particulate matter that have the ability to induce oxidative stress in endothelial cells, which raised levels of mRNA in antioxidant enzymes such as hemoglobin oxygenase-1, glutathione transferase, and NADPH dehydrogenase. These are associated with cardiovascular disease by altering endothelial function. Huang also observed that lipid peroxidation damage in human bronchial epithelial cells (BEAS-2B) was related to elemental carbon and organic carbon in particulate matter.

\section{Mutagenic and Carcinogenic Toxicity}

In recent years, the incidence of lung cancer has increased year by year, and the atmospheric PM 2.5 pollution has become more and more serious. PM 2.5 has been confirmed to contain more heavy metals and polycyclic aromatic hydrocarbons. It is speculated that PM 2.5 plays an important role in lung cancer. Hornberg found that PM 2.5 causes a pronounced SCE production in BEAS-2B cells, which suggests that PM 2.5 has genotoxic effects, and the same conclusion has been demonstrated in lung epithelial cells (A549). With the increase of the dosage of PM 2.5 organic extracts, the micronucleus rate of Balb / c373 cells increased significantly, and obvious DNA damage was observed. The same dose of inorganic extracts showed no damage, which indicates that the genotoxicity of PM 2.5 was mainly caused by organic extracts. In the organic extracts of fine particles, many studies have proved that the weak polar component containing nitro PAH has the strongest mutagenic and carcinogenic effects, and it is the main genotoxic agent.

The mechanism of PM 2.5 in lung carcinogenesis is that small particles can inhibit the cytotoxicity of tumor cells by immune reaction, and increase the susceptibility of human to lung cancer; on the other hand, as carriers, harmful substances are brought into the lower respiratory tract to induce production of reactive oxygen species (ROS) and activate the signal transduction pathway, which results in sudden 
change of bronchial epithelial cells. Shi found that fine particles can make the alveolar cells to produce $\mathrm{OH}$ radicals and induce 8-OH desoxyguanosine (8-OhdG) formation. 8-OhdG is a key factor in the formation of tumors, which suggests that fine particles can induce lung cancer induced by oxidative stress. It has been reported that exposure of lung epithelial cells to fine particulate suspensions leads to an increase in the activity of the C Jun protein kinase, an increase in phosphorylated C-Jun reactive protein levels and an increase in AP-1 dependent gene transcription activity. AP-1 may be involved in many gene enhancer associated with cell proliferation and transformation, resulting in increased gene expression. Activation of AP-1 may be one of the potential mechanisms of carcinogenesis of particulate matter. It can be seen that fine particles contain organic compounds acting on respiratory epithelial cells, activate signal transduction, activate oncogene, regulate the expression of genes and tumor suppressor genes, and increase the risk of carcinogenesis.

\section{Summary}

The negative effects of the respiratory system caused by haze are manifold and can not be ignored. Strengthen the atmospheric particulate matter especially the basic research of PM 2.5, such as epidemiology, toxicology research. It can provide effective theoretical assistance to the formulation of ambient air quality standards and control of air particulate pollution, so that to prevent the sudden significant public health incidents caused by air quality deterioration. I believe that in the near future, with the increase of PM 2.5 observation and research, PM 2.5 pathogenicity research will continue to make new breakthroughs.

\section{Acknowledgement}

Research Foundation of Shaanxi xueqian Normal College(Number: 2017YBRS046)

\section{References}

[1] Qi Jingchen. Effects of Haze on Human Health[J]. Chinese Journal of Urban and Rural Health, 2017, (03): 43-47.

[2] Zheng Jingyuan, Hao Xinying, Wang Pei, Kong Xiangjiu, Chen Qixian. Influence of Haze on People's Outdoor Sports and Countermeasures[J]. Modern Sports Science and Technology, 2016, (14): $141+143$.

[3] Li Ying. Effects of Haze on Respiratory Diseases[J]. The Latest World Medical Information Digest, 2015, (29): 174.

[4] Liu Jianqiu, Chen Xuejiao, Li Zhuying. Effects of Haze on Respiratory Diseases[J]. Chinese Emergency of Chinese Medicine, 2015, (06): 1015-1017.

[5], Xing Lin, Wang Wei, Yu Yang, Yu Lijun. Effects of Haze on Respiratory and Cardiovascular Diseases in the Elderly[J]. Occupation and Health, 2014, (16): 2277-2279.

[6] Health Impacts of Haze[J]. Chinese Journal of Endemic Diseases Control, 2013, (06): 415.

[7] Chen Renjie, Kan Haidong. Haze Pollution and Human Health[J]. Journal of Nature, 2013, (05): 342-344. 